ISSN 0103-5150

Fisioter. Mov., Curitiba, v. 24, n. 1, p. 107-114, jan./mar. 2011 Licenciado sob uma Licença Creative Commons

\title{
Relação da medida da amplitude tóraco-abdominal de adolescentes asmáticos e saudáveis com seu desempenho físico
}

\author{
Relationship of the measure of the amplitude thoracoabdominal in \\ asthmatics and healthy adolescents with the physical performance
}

\author{
Renata Pedrolongo Basso ${ }^{[a]}$, Eloisa Maria Gatti Regueiro ${ }^{[\mathrm{b}]}$, Mauricio Jamami ${ }^{[c]}$, \\ Valéria Amorim Pires Di Lorenzo ${ }^{[\mathrm{d}]}$, Dirceu Costa ${ }^{[\mathrm{e}]}$ \\ [a] Doutoranda do Programa de Pós-Graduação em Fisioterapia da Universidade Federal de São Carlos (UFSCar), São Carlos, \\ SP - Brasil, e-mail: renata.fisio@gmail.com \\ [b] Doutoranda do Programa de Pós-Graduação em Fisioterapia da Universidade Federal de São Carlos (UFSCar), São Carlos, \\ SP - Brasil. \\ [c] Professor Doutor adjunto do curso de Graduação em Fisioterapia e do Programa de Pós-Graduação em Fisioterapia da \\ Universidade Federal de São Carlos (UFSCar), São Carlos, SP - Brasil, e-mail: jamami@ufscar.br \\ [d] Professora Doutora adjunto do curso de Graduação em Fisioterapia e do Programa de Pós-Graduação em Fisioterapia da \\ Universidade Federal de São Carlos (UFSCar), São Carlos, SP - Brasil, e-mail: vallorenzo@ufscar.br \\ [e] Professor Doutor do curso de Pós-Graduação em Fisioterapia da Universidade Nove de Julho (UNINOVE), São Paulo, SP - \\ Brasil, e-mail: dcosta@uninove.br
}

\section{Resumo}

Objetivos: Este estudo teve como objetivo verificar as diferenças das amplitudes tóraco-abdominais nos três níveis (axilar, xifoide e abdominal), nos adolescentes asmáticos e nos saudáveis, e compará-las entre eles. Além de relacioná-las com a capacidade de exercício físico. Materiais e métodos: Foram avaliados 38 adolescentes, na faixa etária dos 11 aos 15 anos, de ambos os sexos, sendo 19 adolescentes asmáticos, que compuseram o grupo asmático (GA), e 19 saudáveis, que compuseram o grupo saudável (GS). Foi preenchida uma ficha de anamnese e realizada a avaliação antropométrica, espirométrica, o teste do degrau de seis minutos (TD6) e a cirtometria, e calculado o índice de amplitude tóraco-abdominal (IA) Resultados: Observou-se diferença significativa para IA entre os níveis axilar e abdominal e xifoidiano e abdominal em ambos os grupos. Não houve diferença significativa quanto ao IA entre os grupos. Somente no GA houve correlação positiva do número total de subidas no TD6 com o IA axilar e xifoide. Conclusão: Para adolescentes 
com asma intermitente e persistente leve não houve diferença na cirtometria tóraco-abdominal quando comparados aos saudáveis e houve correlação dos IA axilar e xifoidiano com o desempenho físico no TD6.

Palavras-chave: Asma. Exercício físico. Adolescentes.

\section{Abstract}

Aims: The aims were verify the differences of amplitude thoracoabdominal by cirtometry, at the levels axillary, xiphoid and abdominal in asthmatics and healthy adolescents and to compare the difference between them. In addition correlate them with the physical exercise capacity. Materials and methods: Thirty eight adolescents were assessed, aged from 11 to 15 years, of both sex. Nineteen were asthmatic adolescent, which composed the asthmatic group ( $A G)$, and nineteen were health adolescents, which composed the healthy group (HG). A clinical history was provided and all subjects were submitted an anthropometric and spirometric evaluation, the six minute step test (6MST), the cirtometry, and was calculated the amplitude thoracoabdominal index (TAI). Results: Significant difference ( $p<0.05)$ was observed to TAI, between axillary and abdominal levels, and xiphoid and abdominal levels in both group. No significant difference in regard to TAI between groups. Only in the AG there was positive correlation of the total number of ascent in the 6MST with the axillary and xiphoid TAI ( $r=0,57$ e $r=0,50 ; p<0,05)$. Conclusion: There were no difference in the thoracic and abdominal cirtometry in adolescents with intermittent and persistent mild asthma when compared with the healthy adolescents and there were correlation axillary and xiphoid TAI with the physical performance in the 6MST.

Keywords: Asthma. Exercise. Adolescent.

\section{Introdução}

A asma é uma doença inflamatória crônica, caracterizada por hiper-responsividade das vias aéreas inferiores e por limitação variável do fluxo aéreo, reversível espontaneamente ou com tratamento, manifestando-se clinicamente por episódios recorrentes de sibilância, dispneia, aperto no peito e tosse, particularmente à noite e pela manhã ao despertar. Resulta da interação entre genética, exposição ambiental a alérgenos e irritantes e outros fatores específicos que levam ao desenvolvimento e à manutenção dos sintomas (1-3).

Durante a crise de asma, o estreitamento da via aérea resulta em aumento da resistência ao fluxo de ar, aprisionamento aéreo e uso excessivo da musculatura inspiratória acessória, o que leva a um padrão ventilatório apical. Com o tempo, dependendo da frequência e da gravidade das crises, esse aumento do trabalho muscular causa alterações na caixa torácica e na postura (4), pois o uso excessivo dessa musculatura leva ao seu encurtamento e com isso o tórax fica em posição de hiperinsuflação, prejudicando a ação mecânica do músculo diafragma e alterando o posicionamento da coluna cervical e da cintura escapular, resultando em uma alteração postural global (5).
Isso pode refletir em medidas alteradas da amplitude tóraco-abdominal nos asmáticos em relação aos saudáveis e também prejudicar sua capacidade de realizar exercícios físicos, pois diminui a capacidade ventilatória, piorando a sensação de dispneia, o que determina menor tolerância ao exercício físico se comparado ao não asmático $(6,7)$.

Dentre as medidas das amplitudes de movimento torácico e abdominal, a cirtometria ou a perimetria tóraco-abdominal têm sido utilizadas na avaliação de doenças respiratórias (8-12), no pré e pós-intervenções terapêuticas (13-16) e em pós-cirúrgicos $(17,18)$, pois a partir dessas medidas observam-se valores de expansibilidade do tórax e do abdômen, de forma simples e acessível, permitindo, assim, auxiliar na identificação do possível problema, possibilitando melhor planejamento do tratamento (19, 20). Considerando as alterações na caixa torácica e o aumento do trabalho muscular que os adolescentes asmáticos apresentam, há carência de estudos e de resultados sobre as evidências da relação da amplitude tóraco-abdominal com a capacidade de exercício físico, sobretudo em adolescentes asmáticos.

Diante disso, o objetivo deste estudo foi verificar as diferenças das amplitudes tóraco-abdominais nos três níveis (axilar, xifoide e abdominal), nos 
adolescentes asmáticos e nos saudáveis, e comparálas entre eles. Além disso, objetivou-se relacioná-las com a capacidade de exercício físico.

\section{Materiais e métodos}

Este estudo foi realizado na Unidade Especial de Fisioterapia Respiratória (UEFR) da instituição e em uma escola pública estadual do município de São Carlos, SP, nas quais foram avaliados 38 adolescentes, na faixa etária dos 11 aos 15 anos, de ambos os sexos, sendo 19 adolescentes asmáticos, que compuseram o grupo asmático (GA), e 19 saudáveis, que compuseram o grupo saudável (GS). Os pais ou responsáveis de todos os adolescentes assinaram o Termo Formal de Consentimento Livre e Esclarecido e o estudo foi aprovado pelo Comitê de Ética e Pesquisa da instituição em que o estudo foi desenvolvido (Parecer n. 119/2008), segundo Resolução 196/96 do Conselho Nacional de Saúde.

\section{Critérios de inclusão e exclusão}

No GA foram incluídos adolescentes com diagnóstico clínico e/ou espirométrico de asma, confirmado por meio da espirometria pré e pós-broncodilatador (BD) realizada sob a supervisão de um médico pneumologista (21); clinicamente estáveis; sem relato de infecções respiratórias e crises por um período mínimo de três semanas. No GS foram incluídos adolescentes sem história de doença respiratória, verificada pelo questionário International Study of Asthma and Allergies in Childhood (ISAAC) de asma e rinite alérgica $(22,23)$, e que apresentaram função ventilatória dentro dos padrões de normalidade constatada pela espirometria. Foram excluídos de ambos os grupos aqueles que apresentaram outras doenças respiratórias, cardíacas, reumáticas, osteomusculares, ortopédicas e sequelas neurológicas associadas que os impedissem de realizar a avaliação proposta.

\section{Procedimento experimental}

Tanto no âmbito escolar como na UEFR, os adolescentes foram primeiramente submetidos a uma entrevista, na qual foi preenchida uma ficha de anamnese e realizada a avaliação antropométrica, a espirométrica, da capacidade ao exercício e a cirtometria.

\section{Avaliação antropométrica e espirométrica}

Consistiu na mensuração da altura (m) e do peso $(\mathrm{kg})$ por uma balança antropométrica $\left(\right.$ Welmy ${ }^{\circledR}$, modelo 110FF, São Paulo, SP, Brasil), tendo os adolescentes descalços, vestidos com bermuda e camiseta. Com essas medidas foi calculado o índice de massa corpórea $\left(\right.$ IMC $=$ peso $/$ altura $^{2}$ ) e foi realizada a classificação de acordo com as curvas de percentil, segundo o gênero e a idade, em desnutridos $<5$, com peso normal $5 \leq \mathrm{x}<85$, sobrepeso $85 \leq \mathrm{x}<95 \mathrm{e}$ obesos $x \geq 95(24,25)$.

Em seguida, tanto asmáticos quanto saudáveis foram submetidos a uma espirometria, com um espirômetro portátil (EasyOne ${ }^{\circledR}$, ndd, Zurique, Suíça), a fim de verificar a função ventilatória de ambos os grupos, e os procedimentos técnicos, critérios de aceitabilidade e reprodutibilidade seguiram as normas da ATS/ERS (26). Foram obtidas três curvas tecnicamente aceitáveis da capacidade vital lenta (CVL), da capacidade vital forçada (CVF) e da ventilação voluntária máxima (VVM). Os valores obtidos foram comparados aos previstos por Polgar e Promadhat (27).

\section{Avaliação da capacidade de exercício físico}

Teste do degrau de seis minutos (TD6)

0 TD6 foi realizado em um degrau de $20 \mathrm{~cm}$, ou $25 \mathrm{~cm}$ de altura, definido de acordo com a altura do adolescente. Para aqueles com até $1,37 \mathrm{~m}$, foi utilizado o degrau de $20 \mathrm{~cm}$ e naqueles com alturas superiores foi utilizado o de $25 \mathrm{~cm}$, baseado no que foi proposto por Feinstein et al. (28), por causa da segurança e para padronizar o esforço muscular requerido no teste. 0 teste seguiu os mesmos princípios da ATS (29) para o TC6 e a cadência de execução foi livre de acordo com Dal Corso et al. (30). O desempenho físico no teste foi determinado pelo número total de subidas no degrau (TD6-T).

Durante o teste, a cada minuto o indivíduo recebia frases de incentivo preestabelecidas e a cada dois minutos foram verificados os sintomas de dispneia e fadiga dos membros inferiores (MMII), por 
meio da escala CR10 de BORG, a saturação periférica de oxigênio $\left(\mathrm{SpO}_{2}\right)$, com um oxímetro de pulso (Nonin ${ }^{\circledR}$, modelo 2500 , Minneapolis, Mn, EUA); e a frequência cardíaca ( $F C$ ), por meio de um frequencímetro (Polar Vantage NV ${ }^{\mathrm{T}}$, modelo 1901001, Finlândia) (31). Essas medidas associadas à pressão arterial sistólica (PAS) e diastólica (PAD), obtida por meio de um esfigmomanômetro $\left(\mathrm{BD}^{\circledR}{ }^{\circledR}\right.$, Curitiba, $\mathrm{PR}$, Brasil) e um estetoscópio (Littmann ${ }^{\circledR}$, modelo classic II S.E., Oakdale, MN, EUA) e da frequência respiratória (FR), foram verificadas no repouso, imediatamente após o teste e no primeiro, no terceiro e no sexto minutos da recuperação.

\section{Avaliação da mobilidade da caixa torácica}

Foi realizada sempre pelo mesmo examinador, por meio da cirtometria, utilizando-se uma fita métrica, escalonada em centímetros ( $\mathrm{cm}$ ) e colocada horizontalmente em três níveis: axilar, xifoidiano e abdominal. Com o indivíduo em posição ortostática e o tórax desnudo, a fita métrica, na região axilar, foi colocada logo abaixo da prega axilar, tomando-se o cuidado para que ficasse firmemente posicionada em linha reta e horizontal. Em seguida, de maneira semelhante, foi posicionada na região xifoidiana, tomando-se como ponto de referência a borda inferior do apêndice xifoide e, por último, foi posicionada na região abdominal, sobre a cicatriz umbilical. Em cada um desses níveis, o indivíduo foi orientado a realizar uma inspiração máxima e logo após uma expiração máxima, sem direcionar o ar para uma ou outra região específica, deixando a fita percorrer os dedos do avaliador durante as manobras, sem deixá-la com folgas ou apertada demais. Foram solicitadas que essas manobras fossem repetidas mais duas vezes, sendo anotados os três valores máximos de inspiração e expiração (19). Entretanto, para a análise dos dados foi considerado o maior valor obtido das três medidas realizadas e calculado o Índice de Amplitude (IA), proposto por Jamami et al. (13), com a finalidade de atenuar as diferentes dimensões de tórax e abdômen para a amostra estudada, por meio da seguinte fórmula:

$$
\mathrm{IA}=\left(\frac{\frac{\mathrm{INS}-\mathrm{EXP}}{\mathrm{INS}}+\frac{\mathrm{INS}-\mathrm{EXP}}{\mathrm{EXP}}}{2}\right) \times 100
$$

\section{Análise estatística}

Foi realizado o teste de Shapiro-Wilk, a fim de verificar a normalidade na distribuição dos dados. A estatística descritiva foi realizada para a caracterização da amostra, sendo os dados expressos em média \pm desvio-padrão. Para a análise intergrupos foi utilizado o teste $t$ de Student independente e, para a análise intragrupo, o teste $t$ de Student pareado, além do coeficiente de correlação de Pearson. 0 programa estatístico utilizado foi o Statistical Package for the Social Sciences (SPSS) para Windows, versão 13.0. 0 nível de significância adotado foi de $5 \%$.

\section{Resultados}

Os adolescentes asmáticos foram classificados em asma intermitente e persistente leve, de acordo com a Global Initiative for Asthma (21). Cinco desses adolescentes faziam uso regular de corticosteroide inalatório (dose média de $200 \mathrm{mcg}$ ) e os demais uso de beta-2 agonista quando necessário para o alívio dos sintomas. Quanto à classificação do IMC no GA havia: 13 com peso normal, 2 com sobrepeso e 4 obesos, já no GS havia: 12 com peso normal, 5 com sobrepeso e 2 obesos.

As características antropométricas e espirométricas de cada grupo estão demonstradas na Tabela 1. $\mathrm{Na}$ análise intergrupos verificou-se diferença significativa somente para a VVM \% do predito (VVM\% pred) ( $p=0,003)$, visto que a média foi maior no GS.

Quanto ao TD6, observou-se diferença significativa quanto ao TD6-T ( $\mathrm{p}=0,005)$, visto que a média foi maior no GS (Tabela 1). Vale ressaltar que três indivíduos do GA interromperam o TD6 por cerca de 20 segundos em média, em razão da fadiga de MMII, e depois retomaram até completar os seis minutos do teste.

Em relação aos valores da cirtometria não houve diferença significativa quanto ao IA entre os grupos (Tabela 2).

$\mathrm{Na}$ análise intragrupo para as medidas de cirtometria, observou-se diferença significativa para IA, entre os níveis axilar e abdominal e xifoidiano e abdominal, em ambos os grupos (Tabela 2).

Quanto à correlação da capacidade de exercício físico com as medidas da cirtometria, observou-se que somente no GA houve correlação positiva do TD6-T com o IA axilar e xifoide (Tabela 3). 
Tabela 1 - Variáveis antropométricas, espirométricas e 0 desempenho físico nos grupos asmático (GA) e saudável (GS)

\begin{tabular}{|c|c|c|}
\hline & $\begin{array}{c}\text { GA } \\
(n=19)\end{array}$ & $\begin{array}{c}\text { GS } \\
(n=19)\end{array}$ \\
\hline $\operatorname{Sexo}(h / m)$ & $15 / 4$ & $13 / 6$ \\
\hline Idade (anos) & $12 \pm 1,4$ & $12 \pm 1,0$ \\
\hline Peso (kg) & $52 \pm 14,1$ & $46 \pm 9,8$ \\
\hline Altura (m) & $1,6 \pm 0,1$ & $1,5 \pm 0,9$ \\
\hline IMC (kg/m²) & $21 \pm 4,1$ & $21 \pm 4,8$ \\
\hline $\operatorname{VEF}_{1}(\mathrm{~L})$ & $2,5 \pm 0,6$ & $2,3 \pm 0,3$ \\
\hline $\operatorname{VEF}_{1}(\%$ pred $)$ & $88 \pm 8,1$ & $93 \pm 11,6$ \\
\hline CVF (L) & $2,9 \pm 0,6$ & $2,7 \pm 0,4$ \\
\hline CVF (\% pred) & $93 \pm 6,5$ & $97 \pm 12,5$ \\
\hline $\mathrm{VEF}_{1} / \mathrm{CVF}(\mathrm{L})$ & $0,9 \pm 0,1$ & $0,9 \pm 0,5$ \\
\hline $\mathrm{VEF}_{1} / \mathrm{CVF}$ (\% pred) & $93 \pm 7,5$ & $95 \pm 4,7$ \\
\hline VVM (L/min) & $78 \pm 20,6$ & $86 \pm 16,1$ \\
\hline VVM (\% pred) & $80 \pm 13,9$ & $96 \pm 13,1^{*}$ \\
\hline TD6-T $\left(n^{0}\right)$ & $147 \pm 30,4$ & $171 \pm 14,1^{*}$ \\
\hline
\end{tabular}

Obs.: os dados estão expressos em média \pm desvio-padrão. Legenda: IMC = índice de massa corpórea; VEF $_{1}=$ volume expiratório forçado no primeiro segundo; CVF = capacidade vital forçada; $V_{E F} / C V F=$ relação VEF, $/ C V F ; V V M=$ ventilação voluntária máxima; TD6-T = número total de subidas no teste do degrau de seis minutos; ${ }^{*}=$ teste $t$ independente $(p \leq 0,05)$.

\section{Discussão}

A cirtometria faz parte da avaliação fisioterapêutica e visa a quantificar as medidas de mobilidade tóraco-abdominal. Estudos mostram ser uma medida confiável, intra e interexaminadores, para medir as amplitudes dos movimentos toracoabdominais, porém, não é uma medida acurada para medir os volumes pulmonares, não devendo ser utilizada para esse fim na prática clínica ou na pesquisa científica $(12,19,20)$.

Diante disso, optou-se neste estudo por não se utilizar para análise os valores absolutos de perímetro do tórax, mas sim os valores do índice de amplitude (IA), para que dessa forma as diferentes dimensões do tórax e do abdômen, em virtude das constituições antropométricas individuais, não interferissem na análise dos dados de amplitude.
Tabela 2 - Índice de amplitude (IA) nos grupos asmático (GA) e saudável (GS)

\begin{tabular}{lrl}
\hline & GA & GS \\
\hline IA axilar & $5,9 \pm 3,2$ & $7,2 \pm 2,8$ \\
IA xifoidiano & $5,2 \pm 2,9$ & $6,9 \pm 2,9$ \\
IA abdominal & $-0,2 \pm 2,7^{\star}$ & $0,6 \pm 4,8^{\star}$ \\
\hline
\end{tabular}

Obs.: análise intragrupo (interníveis): * teste t pareado $(p \leq 0,05)$.

Tabela 3 - Correlação do índice de amplitude (IA) com TD6 no grupo asmático (GA)

\begin{tabular}{lcc}
\hline & \multicolumn{2}{c}{ TD6-T } \\
\cline { 2 - 3 } & $\mathbf{r}$ & $\mathbf{p}$ \\
\hline IA axilar & $0,57^{\star}$ & 0,01 \\
IA xifoidiano & $0,50^{*}$ & 0,03 \\
IA abdominal & 0,47 & 0,85 \\
\hline
\end{tabular}

Legenda: TD6-T = número total de subidas no teste do degrau de seis minutos; $r=$ coeficiente de correlação de Pearson.

Quanto às medidas do IA, observou-se que entre os grupos os valores do GA tenderam a ser menores, porém, não foram encontradas diferenças significativas. Além disso, os valores de amplitude mostraram-se decrescentes do nível axilar ao abdominal, assim como citado por outros autores $(19,20)$, sendo isso evidenciado pela diferença significativa para as medidas do nível abdominal em relação ao nível axilar e xifoidiano. Os valores abdominais, por sua vez, foram negativos no GA, o que mostra que no momento da medida, ao se pedir uma inspiração profunda aos adolescentes asmáticos, eles realizaram, de um modo geral, um padrão respiratório paradoxal, ou seja, retraindo o abdômen no momento da inspiração. Apesar da cirtometria não avaliar padrão respiratório, isso ressalta que o padrão diafragmático não é algo usual para esses adolescentes, e em um momento de aumento da demanda ventilatória possivelmente o padrão apical se sobressaia.

Apesar de não haver valores de normalidade para o IA, alguns autores consideram que valores de amplitude tóraco-abdominal normais para adultos jovens seriam em torno de $4-7 \mathrm{~cm}(32,33)$. No presente estudo, os valores para os níveis axilar e 
xifoidiano ficaram dentro dessa faixa, tanto para os adolescentes asmáticos como para os saudáveis.

Isso mostra que os adolescentes asmáticos deste estudo não apresentaram déficit de mobilidade torácica, o que pode ter ocorrido pelo fato da asma ter sido classificada como intermitente e persistente leve, uma vez que tanto a frequência como a gravidade das crises são os fatores que determinam as alterações na conformação do tórax e na possível diminuição da mobilidade torácica (4).

Quanto à capacidade para o exercício físico, optou-se por realizar o TD6, por ser um teste que vem sendo descrito na literatura na avaliação de pneumopatas $(28,30)$ e por se tratar de um teste submáximo, que avalia a capacidade funcional do indivíduo na realização de suas atividades de vida diária (30), além de ser de fácil aplicação, seguro e acessível.

Observou-se que o desempenho físico no teste (TD6-T) foi significativamente menor no GA e que para esse grupo houve correlação positiva entre os IA axilar e xifoidiano com o TD6-T, o que mostra que, para o adolescente asmático, quanto maior a mobilidade torácica melhor seu desempenho no TD6.

Esse resultado sugere que uma melhor mobilidade torácica implicaria melhor ventilação pulmonar e com isso diminuição da dispneia (15). Uma vez que não há na literatura estudos com esse enfoque e para essa população, isso foi baseado em estudos, com doentes pulmonares obstrutivos crônicos, que mostram que exercícios físicos voltados para a mobilidade do tórax melhoram a ventilação, a capacidade submáxima ao exercício e reduzem a dispneia $(15,34)$.

Pensando nisso, ressalta-se a importância de se ter medidas de normalidade para a amplitude tóraco-abdominal para adolescentes saudáveis e, assim, ter um parâmetro adequado de avaliação em asmáticos, a fim de planejar uma terapêutica voltada não somente para o condicionamento físico, mas também para reeducação diafragmática e para a mobilidade do tórax e do abdômen.

Neste estudo, algumas limitações podem ser observadas, como a necessidade de uma amostra maior e com maior diversidade da classificação da asma, para que se possa identificar se naqueles mais graves há diminuição da mobilidade tóraco-abdominal, além da identificação durante o TD6 das medidas de ventilação e do padrão respiratório adotado pelos adolescentes asmáticos, para confirmar se a melhor ventilação durante o teste está relacionada com a maior mobilidade tóraco-abdominal.

\section{Conclusão}

0 estudo mostrou que, para adolescentes com asma intermitente e persistente leve, não houve diferença na cirtometria tóraco-abdominal, quando comparados aos jovens saudáveis, e houve correlação dos IA axilar e xifoidiano com o desempenho físico no TD6.

\section{Referências}

1. Sociedade Brasileira de Pneumologia e Tisiologia. IV Diretrizes Brasileiras para o Manejo da Asma. J Bras Pneumol. 2006;32(Suppl 7):S447-S74.

2. Busse WW, Lemanske RF. Asthma. N Engl J Med. 2001; 344(5):350-62.

3. Cookson W. The alliance of genes and environment in asthma and allergy. Nature. 1999;402(6760 Suppl): B5-11.

4. Lopes EA, Fanelli-Galvani A, Prisco CCV, Gonçalves RC, Jacob CMA, Cabral ALB, et al. Assessment of muscle shortening and static posture in children with persistent asthma. Eur J Pediatr. 2007;166(7):715-21.

5. Pasinato F, Corrêa ECR, Peroni ABF. Avaliação da mecânica ventilatória em indivíduos com disfunção têmporo-mandibular e assintomáticos. Rev Bras Fisioter. 2006;10(3):285-89.

6. Priftis KN, Panagiotakos DB, Anthracopoulos MB, Papadimitriou A, Nicolaidou P. Aims, methods and preliminary findings of the physical activity, nutrition and allergies in children examined in Athens (PANACEA) epidemiological study. BMC Public Health. 2007;7(140):1-23.

7. Pianosi PT, Davis HS. Determinants of physical fitness in children with asthma. Pediatrics. 2004;113(3): 225-29.

8. Ringel ER, Loring SH, McFadden Jr ER, Ingram Jr RH. Chest wall configurational changes before and during acute obstructive episodes in asthma. Am Rev Respir Dis. 1983;128(4):607-10.

9. Maciel SS, Paulo MQ Souza CQ, Silva LG, Tavares RR. Efeitos do broncodilatador do Acanthospermum hispidum DC, nos pacientes pulmonares obstrutivos crônicos (DPOC). Rev Bras Ciênc Saúde. 1997; 1(1/3):23-30. 
10. Caromano FA, Durigon OFS, Landaburu C, Pardo MS. Estudo comparativo de duas técnicas de avaliação da mobilidade torácica de mulheres jovens e idosas saudáveis. Fisioter Bras. 2003;4(5):348-52.

11. Silva FB, Sampaio LMM, Carrascosa AC. Avaliação fisioterapêutica dos sistemas mastigatório e respiratório de um portador de síndrome otodental: um estudo de caso. Rev Bras Fisioter. 2006;10(1):133-36.

12. Malaguti C, Rondelli RR, Souza LM, Domingues M, Dal Corso S. Reliability of chest wall mobility and its correlation with pulmonary function in patients with chronic obstructive pulmonary disease. Respir Care. 2009;54(12):1703-11.

13. Jamami M, Pires VA, Oishi J, Costa D. Efeitos da intervenção fisioterápica na reabilitação pulmonar de pacientes com doença pulmonar obstrutiva crônica (DPOC). Rev Fisioter Univ São Paulo. 1999; 6(2):140-53.

14. Costa D, Sampaio LMM, Di Lorenzo VAP, Jamami M, Damaso AR. Avaliação da força muscular respiratória e amplitudes torácicas e abdominais após RFR em indivíduos obesos. Rev Latino-Am J Nursing. 2003; 11(6):156-60.

15. Paulin E, Brunetto AF, Carvalho CRF. Efeitos de programa de exercícios físicos direcionado ao aumento da mobilidade torácica em pacientes portadores de doença pulmonar obstrutiva crônica. J Pneumol. 2003;29(5):287-94.

16. Moreno MA, Catai AM, Teodori RM, Borges BLA, Cesar MC, Silva E. Efeito de um programa de alongamento muscular pelo método de reeducação postural global sobre a força muscular respiratória e a mobilidade toracoabdominal de homens jovens sedentários. J Bras Pneumol. 2007;33(6):679-86.

17. Garcia RCP, Costa D. Treinamento muscular respiratório em pós-operatório de cirurgia cardíaca eletiva. Rev Bras Fisioter. 2002;6(3):139-46.

18. Silva EF, Guedes RP, Ribeiro EC. Estudo das repercussões das cirurgias abdominais sobre os músculos respiratórios. Fisioter Mov. 2003;16(1):51-6.

19. Borghi-Silva A, Mendes RG, Silva ES, Paulucci HL, Picchi PC, Di Lorenzo VAP. Medida da amplitude tóraco-abdominal como método de avaliação dos movimentos do tórax e abdome em indivíduos jovens saudáveis. Fisioter Brasil. 2006;7(1):25-9.
20. Caldeira VS, Starling CCD, Britto RR, Martins JA, Sampaio RF, Parreira VF. Precisão e acurácia da cirtometria em adultos saudáveis. J Bras Pneumol. 2007;33(5):519-26.

21. Global Initiative for Asthma. Global strategy for asthma management and prevention. 2007. [acesso em 26 jan. 2010]. Disponível em: www.ginasthma.org

22. Solé D, Vanna AT, Yamada E, Rizzo MCV, Naspitz CK. International study of asthma and allergies in childhood (ISAAC) written questionnaire: validation of the asthma component among Brazilian children. Invest Allergol Clin Immunol. 1998;8(6):376-82.

23. Vanna AT, Yamada E, Arruda LK, Naspitz CK, Solé D. International study of asthma and allergies in childhood: validation of the rhinitis symptom questionnaire and prevalence of rhinitis in schoolchildren in São Paulo, Brazil. Pediatr Allergy Immunol. 2001;12(2):95-101.

24. NCHS, National Center for Health Statistics/National Center for Chronic Disease Prevention and Health Promotion. 2000 [acesso em 28 jan. 2010]. Disponível em: http://www,cdc,gov/growthcharts

25. Cassol VE, Rizzato TM, Teche SP. Prevalência e gravidade da asma em adolescentes e sua relação com índice de massa corporal. J Pediatr. 2005;81(4):305-9.

26. Miller MR, Hankinson J, Brusasco F, Casaburi R, Coates A, Crapo R, et al. ATS/ERS task force: standardisation of lung function testing. Eur Respir J. 2005;26:319-38.

27. Polgar C, Promadhat V. Pulmonary function testing in children: techniques and standards. Philadelphia, PA: W. B. Sanders; 1971.

28. Feinstein RA, Hains CS, Hemstreet MP, TurnerHenson A, Redden DT, Martin B, et al. A simple "steptest" protocol for indentifying suspected unrecognized exercise-induced asthma (EIA) in children. Allergy and Asthma Proc. 1999;20(3):181-88.

29. American Thoracic Society. ATS statement: guidelines for the six-minute walk test. Am J Respir Crit Care Med. 2002;166:111-17.

30. Dal Corso S, Duarte SR, Neder JA, Malaguti C, Fuccio MB, Castro Pereira CA, et al. A step test to assess exercise-related oxygen desaturation in interstitial lung disease. Eur Respir J. 2007;29(2):330-36. 
31. Borg GA. Psychophysical bases of perceived exertion. Med Sci Sports Exerc. 1982;14(5):377-81.

32. Carvalho A. Semiologia em reabilitação. São Paulo: Atheneu; 1994.

33. Lianza S. Medicina de reabilitação. 2a ed. Rio de Janeiro: Guanabara Koogan; 1995.
34. Kakizaki F, Shibuya M, Yamazaki T, Yamada M, Suzuki H, Homma I. Preliminary report on the effects of respiratory muscle stretch gymnastics on chest wall mobility in patients with chronic obstructive pulmonary disease. Respir Care. 1999;44:409-14.

Recebido: $18 / 07 / 2010$

Received: 07/18/2010

Aprovado: 20/10/2010

Approved: 10/20/2010 